\title{
A microwave doppler radar activity monitor
}

\author{
P. H. MARTIN \\ University Sub-Department of Animal Behaviour \\ Cambridge University, Madingley, Cambridge CB3 $8 A A$, England \\ and \\ D. M. UNWIN \\ Department of Zoology \\ Cambridge University, Downing Street, Cambridge CB2 3EJ, England
}

\begin{abstract}
The general requirements of an activity monitor are listed. The principle of operation of microwave doppler radar is described. The circuitry is given for a sensitive radar activity monitor that works by detecting the doppler shift in microwaves reflected from a moving animal. The apparatus is automatic and nonintrusive, and it gives an output suitable for direct computer analysis. A high level of concordance is found with manual observation. A simple means of calibrating the device is described.
\end{abstract}

This paper describes the circuit for a radar activity monitor that works by detecting the doppler frequency shift in microwaves reflected from a moving object. The apparatus has provided continuous records of activity from domestic cats in large enclosures. The radar method has a number of advantages: It is nonintrusive, the output is suitable for direct computer analysis, and the physical layout can easily be adapted to suit a wide range of applications with animals and enclosures of different sizes. The microwave doppler technique has been used to record activity in small enclosures with insects (Buchan \& Sattelle, 1979) and shrews (Godfrey, 1978), and it is used in some commercial burglar alarms that may be modified for scientific applications (Heal, 1975).

\section{REQUIREMENTS OF AN ACTIVITY MONITOR}

In any technique for recording activity, several features are desirable. (1) The actual behavior that is recorded should be clearly defined, but not too tightly constrained by the nature of the apparatus itself. Running wheels (Richter, 1927), feeding or drinking sensors (Lehmann, 1976), and trapping records (Daan \& Slopsema, 1978) obviously record behavior within the broad category of "activity." Mechanical switches in the floor (Broom, 1980), completion of electrical circuits in a floor matrix (Pfister, Mudge, \& Harcombe, 1978), spring-suspended cages (Aschoff, 1962), and leverbalance cages (Campbell \& Shipp, 1974) have also been used.

(2) The sensitivity of the apparatus should be spa-

This work was supported in part by a Medical Research Council studentship awarded to P. H. Martin. We are very grateful to Christine Proby and Paul Heavens for their assistance and to Michael Simpson, Patrick Bateson, and Robert Hinde for their comments on the draft manuscript. Correspondence should be addressed to D. M. Unwin. tially homogeneous, that is, roughly constant throughout the enclosure. This is difficult to achieve with photoelectric devices (Moross \& Kaufman, 1976), especially in large enclosures.

(3) The technique should be nonintrusive. Here, the radar has a clear advantage over the use of chronically worn inertial sensors (Delgado-Garcia, Grau, De Feudis, Del Pozo, Jimenez, \& Delgado, 1976) and other telemetric methods.

(4) The monitoring should be continuous and automatic, giving long and uninterrupted outputs that are easy to analyze, preferably by computer. Methods such as time-lapse video (Bowden, Kripke, \& Wyborney, 1978) tend to produce records of limited duration that require extensive subsequent analysis.

(5) The apparatus should have a simple, independent means of calibration. This enables the appropriate characteristics of the apparatus to be set up in a repeatable manner; it is also necessary for checking any drift in characteristics with time.

(6) The apparatus should be validated by comparing its output with activity records obtained by some independent means (Anastasi, 1968), preferably by manual recording of observations.

All of these requirements are fulfilled by the radar apparatus described here. However, one limitation of the radar is that it does not distinguish between individuals. Thus, if more than one animal is present in the enclosure, the record is essentially of group activity. There seems to be no simple, nonintrusive way of adapting the method for individual identification at this time.

\section{MICROWAVE DOPPLER RADAR: PRINCIPLE OF OPERATION}

Waves reflected from an object that is moving with respect to the wave detector will be shifted in frequency 
(the doppler effect). In this apparatus, movement is sensed by a microwave doppler radar module, which consists of an emitter and a detector of microwaves and a mixer whose output is a voltage at the difference (beat) frequency. Waves reflected from a stationary object are at the same frequency as the emitted waves, resulting in a mixer output of zero frequency (dc voltage). Waves reflected from a moving object are shifted in frequency, resulting in an ac voltage at the mixer. The frequency of the mixer signal (equal to the doppler shift) is dependent on the relative velocity of the moving reflector, with the approximate relationship given by $f_{D}=2 v / \lambda$, where $f_{D}$ is the doppler shift (mixer output frequency), $v$ is the relative velocity of the reflector, and $\lambda$ is the wavelength. In this case, microwaves of wavelength $2.8 \mathrm{~cm}$ are used, giving a doppler shift of about $71 \mathrm{~Hz} / \mathrm{msec}^{-1}$ of velocity. Thus, with the velocities normally attained by animals, the output of the mixer will be an ac voltage (henceforth referred to as the doppler signal) in the subaudio to audio frequency range.

\section{DESCRIPTION}

\section{Circuit design}

The ac doppler signal from the radar module is amplified by a two-stage, low-noise audio amplifier. The processing of the doppler signal is by a peak detector triggering a monostable multivibrator. When the doppler signal reaches a threshold, the monostable is activated, causing a set of relay contacts to close for a predetermined period (the monostable "dead time"). If the doppler signal remains above threshold, the relay contacts stay closed. This type of processing may be contrasted with the "pulse-counting" mode often used in commercial burglar alarms, in which the circuit operates only after the doppler signal has reached threshold a certain number of times. The overall system design is shown in Figure 1.

The radar module used is a commercially available X-band doppler radar module (Mullard CL8960 or RS RS8960; RS Components Ltd., 13-17 Epworth Street, London EC2P 2HA, England). This system contains a Gunn oscillator that emits microwaves of wavelength $2.8 \mathrm{~cm}$ at a power of $10 \mathrm{~mW}$. The module also contains a mixer diode that combines reflected signals with a sample of the emitted waves to give an ac voltage at the difference frequency (the doppler signal). The antenna provided with the unit gives a wide-angle free-space radiation pattern, described approximately by a 120-deg cone.

The preamplifier (Figure 2) has a gain of about 160 , and the main amplifier (Figure 3) has a variable gain of 7-320. The doppler module and preamplifier are fed from a $7 . \mathrm{V}$ dc regulator (Figure 4), which is in turn supplied from a $12-\mathrm{V} \mathrm{dc}$ main power unit. The design of the amplifiers and the $7-\mathrm{V}$ regulator is taken, with some modifications, from circuits provided by the doppler module supplier.

The amplified doppler signal is fed to the input of a

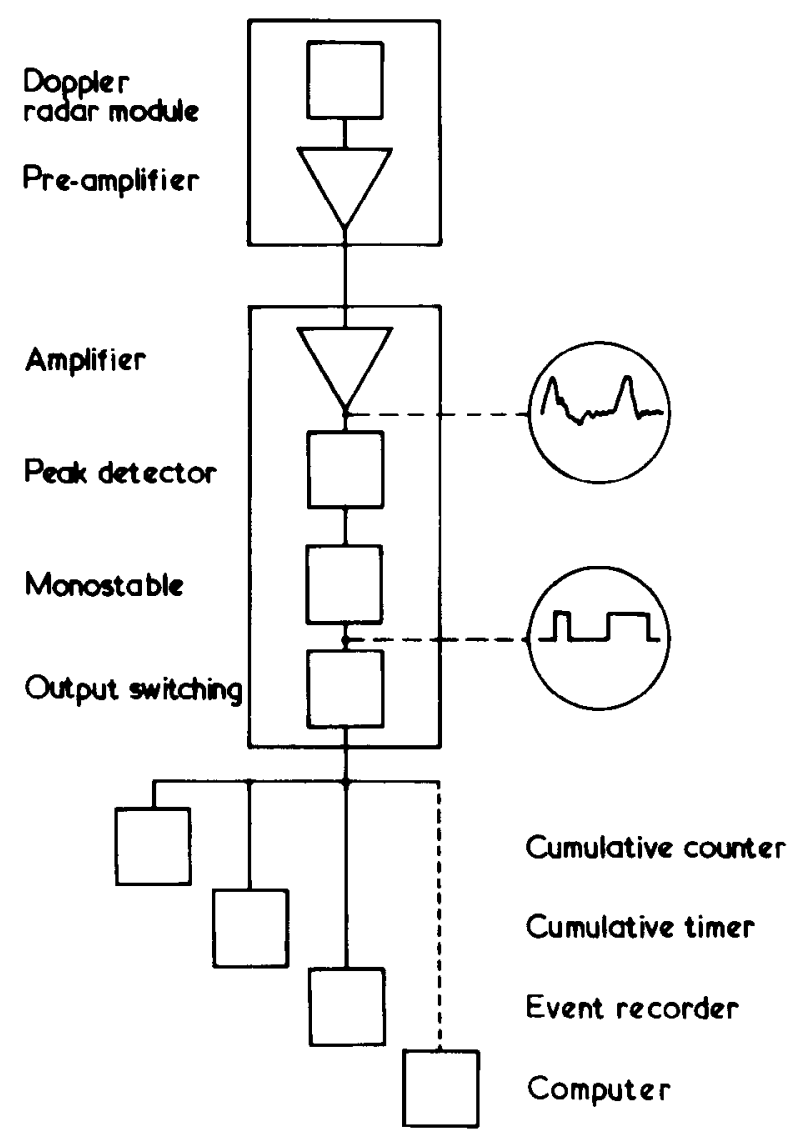

Figure 1. Doppler radar activity monitor: schematic representation of major components and associated recording devices.

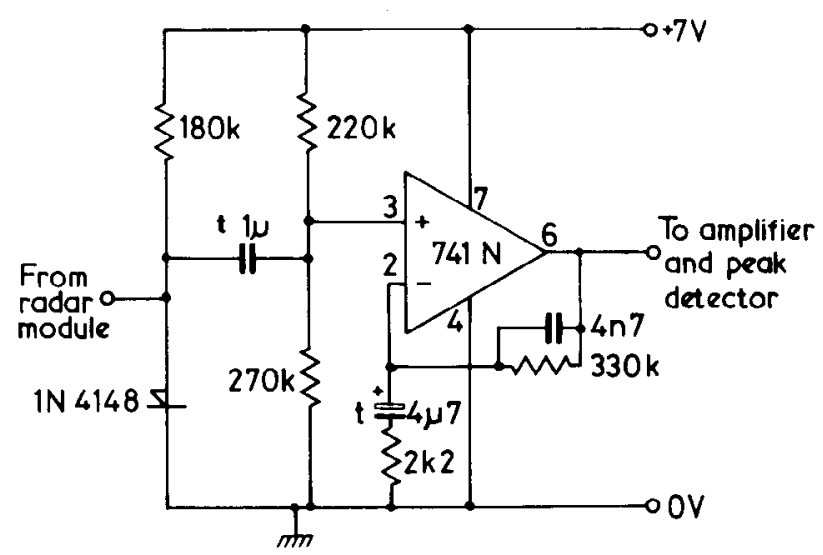

Figure 2. Doppler signal preamplifier ( $T$ denotes tantalum capacitor).

monostable multivibrator (Figure 5), whose dead time (minimum duration of output) is set to $1 \mathrm{sec}$ in the present apparatus. The monostable and main amplifier are fed from a $\pm 15-\mathrm{V}$ dc regulated power supply.

\section{Layout and Construction Notes}

The walls of the enclosure in which the monitor is used should be electrically screened, as far as is practicable, with a conducting material. Aluminum foil or 


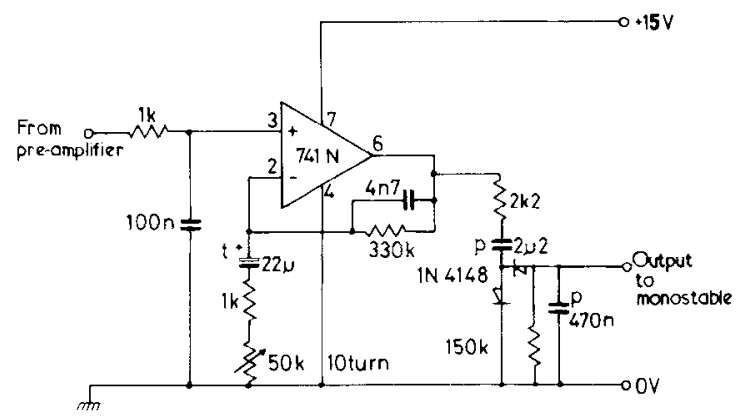

Figure 3. Doppler signal amplifier and peak detector (T denotes tantalum and $\mathbf{P}$ denotes polyester capacitors).

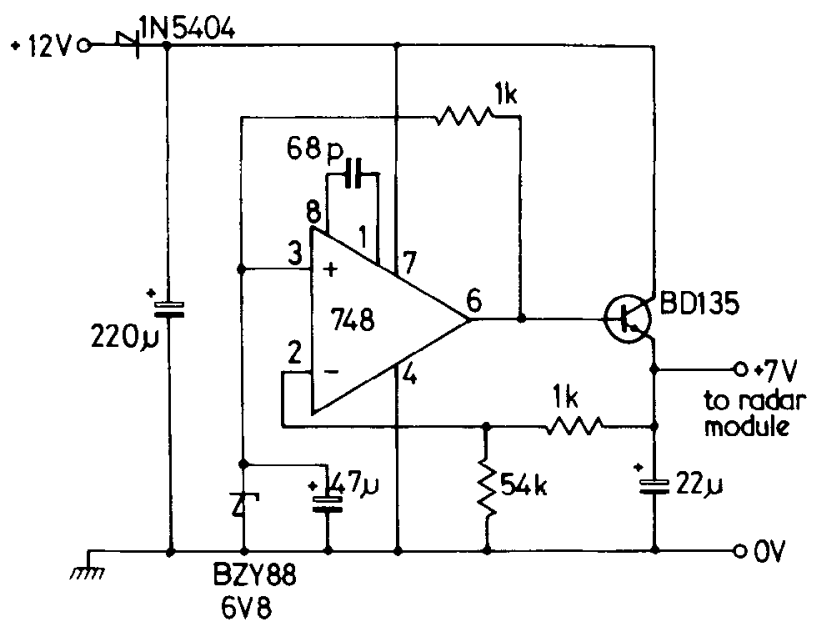

Figure 4. A 7.V dc regulator to supply doppler radar module and preamplifier.

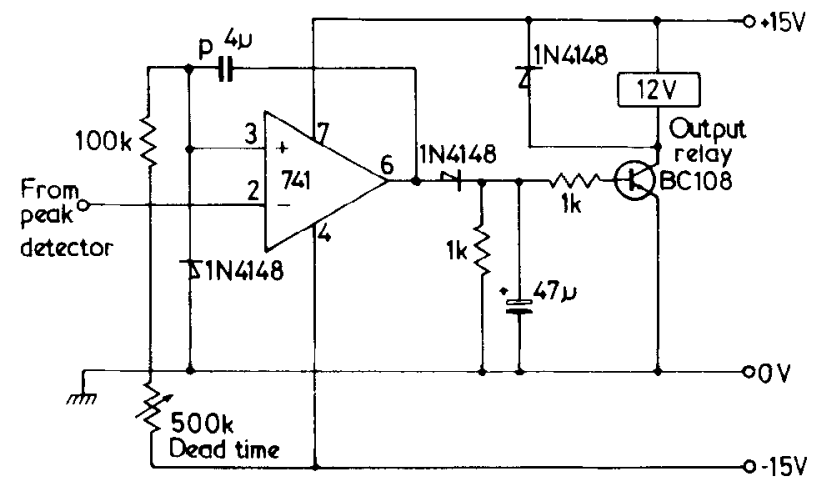

Figure 5. Monostable and output switching circuits.

thin aluminum sheet is both adequate and cheap. Windows may be covered with wire mesh. The screening sets up a standing wave pattern in the enclosure, thus improving the "spatial homogeneity" of the sensitivity; it also prevents spurious triggering of the apparatus by objects moving outside the enclosure.

The apparatus has been used as an activity monitor for domestic cats in a large enclosure (floor area $8.8 \mathrm{~m}^{2}$ ). The radar module, preamplifier, and $7-\mathrm{V}$ regu- lator are housed in a small, grounded metal box mounted over the door to the room, $1.84 \mathrm{~m}$ from the floor. The microwaves are emitted from a small port in the box, which is directed into the center of the room and tilted down at $35 \mathrm{deg}$ to the horizontal. The rest of the apparatus, together with the recording devices, is housed in an adjacent room. The apparatus is potentially sensitive to ac mains noise $(50-100 \mathrm{~Hz})$, so grounded metal boxes and screened interconnecting cables are used throughout. All ac mains supplies are taken from a common source, via a capacitance/inductance filter. Internal noise is reduced by using low-noise integrated circuits (ICs) and thick-film resistors throughout. Some capacitors must be low-leakage types; this is indicated on the circuit diagrams. The ionized gas in ac fluorescent lighting is a modulated reflector of microwaves. It might, therefore, prove undesirable to use such lighting in the enclosure, although no problems have been encountered with the present apparatus.

The activity monitor is relatively cheap and simple to build from readily available components. The parts, excluding power supplies and recording devices, should cost less than $\$ 150$.

\section{Output}

The circuit gives a binary (on-off) output by closing a set of relay switch contacts whenever the doppler signal exceeds a predetermined threshold. In the present set-up, the output is used to operate three recording devices: a cumulative counter, a cumulative timer, and one channel of an electromechanical paper-roll event recorder. The output is in a form suitable for recording directly onto magnetic tape for subsequent computer analysis or for direct input to a dedicated on-line microprocessor. The complete audio-frequency doppler signal is available at the output of the amplifier. By subjecting this signal to spectral analysis or autocorrelation (Buchan \& Sattelle, 1979), it is possible to analyze the velocity components of individual complex movements.

\section{Calibration}

It is necessary, with such a device, to have an independent means of repeatably setting the sensitivity to a predetermined level and of subsequently checking that this setting has not drifted with time. One way of doing this is to feed a sinusoidal ac signal into the amplifier and to measure the ac voltage needed to just trigger the output. The threshold at the input to the main amplifier, which must be dc biased by $4 \mathrm{~V}$, was found to be $11 \mathrm{mV}$ at $10 \mathrm{~Hz}$ and $25 \mathrm{mV}$ at $200 \mathrm{~Hz}$ at the sensitivity setting used.

A more fundamental calibration, which checks all stages of the system, uses a standardized moving object to trigger the apparatus. A small, 60-rpm electric motor is mounted on a length of conduit attached to the ceiling, directly opposite to and $2 \mathrm{~m}$ away from the radar module and $1.5 \mathrm{~m}$ from the floor. Aluminum sheets of different cross-sectional areas can then be attached to 
the motor, with the axis of rotation perpendicular to the floor, to give standard moving "targets" of different radar cross-sections. The sensitivity of the monitor can then be quantified in terms of the target size needed to just trigger the output. The threshold target size was found to be just below $20 \mathrm{~cm}^{2}$ under these conditions. The radar cross-section of a human is about $10,000 \mathrm{~cm}^{2}$ $\left(1 \mathrm{~m}^{2}\right)$; that of an adult cat is about $1,000 \mathrm{~cm}^{2}\left(.1 \mathrm{~m}^{2}\right)$. The reflected power received by the radar varies logarithmically with the target cross-section. In a series of calibration tests carried out over a period of 7 weeks, the threshold was not found to drift significantly, although some small fluctuations may be expected to result from variations in temperature or humidity.

\section{Validation}

A series of tests was carried out to determine the concordance between the radar monitor and manual observation. The activity of the cats was scored by direct observation, and the binary output of the radar was scored simultaneously by a second observer. The two observers were in separate rooms, with no visual or auditory contact, and they alternated roles after each session. Scoring included movements in which translation of the whole body relative to the floor did not necessarily occur.

Both observers were given a common auditory cue through headphones at 6 -sec intervals, and the behavior and radar output were scored on a one-zero basis (i.e., occurrence or nonoccurrence) within each 6-sec block. Observations were in sessions of $10 \mathrm{~min}$ (100 6-sec blocks) duration. A total of 24 sessions ( $4 \mathrm{~h}$ ) of observation was carried out over a 7 -week period, at various times of day and sampling various states of overall activity (including sleep and vigorous play).

A measure of interobserver concordance was obtained, with the radar monitor treated as one observer. The data were partitioned into the same time blocks as those normally used in manual observations in our study, namely, $6 \mathrm{sec}$ and $30 \mathrm{sec}$. Scoring on a one-zero basis in $30-\mathrm{sec}$ blocks (total $=480$ blocks), there were 418 agreements (A) and 62 disagreements (D) between manual observation and the radar, giving a concordance
$[\mathrm{A} /(\mathrm{A}+.5 \mathrm{D})]$ of $.93 . \mathrm{A}$ separate measure of agreement was obtained by summing the "scores" ( 1 or 0 ) for the 6-sec blocks in each 10-min session, giving a score out of 100 for each of the 24 sessions per observer. The product-moment correlation coefficient of the 24 pairs of scores (radar monitor vs. manual observation) was .96 .

\section{REFERENCES}

Anastasi, A. Psychological testing (3rd ed.). New York: Macmillan, 1968.

Aschoff, J. Spontane lokomotorische Aktivitat. Handbuch der Zoologie, 1962, 8, 1-74.

Bowden, D. M., Kripke, D. F., \& Wyborney, V. G. Ultradian rhythms in waking behavior of rhesus monkeys. Physiology \& Behavior, 1978, 21, 929-933.

Broom, D. M. Activity rhythms and position preferences of domestic chicks which can see a moving object. Animal Behav. iour, 1980, 28, 201-211.

Buchan, P. B., \& Satrell.e, D. B. A radar-doppler autocorrelation analysis of insect activity. Physiological Entomology, 1979, 4, 103-109.

CAmpBel.l, D. J., \& Shipr, E. Spectral analysis of cyclic behaviour with examples from the field cricket Teleogryllus commodus (Walk). Animal Behaviour, 1974, 22, 862-875.

DaAn, S., \& Slopsema, S. Short-term rhythms in foraging behaviour of the common vole, Microtus arvalis. Journal of Comparative Physiology, 1978, 127, 215-227

Delgado-Garcia, J. M., Grau, C., De Feudis, P., Del Pozo, F., Jimenez, J. M., \& Deigado, J. M. R. Ultradian rhythms in the mobility and behavior of rhesus monkeys. Experimental Brain Research, 1976, 25, 79-91.

Godfrex, G. K. The activity pattern in white-toothed shrews studied with radar. Acta Theriologica, 1978, 23, 381-390.

HeAL, J. W. An animal activity monitor using a microwave doppler system. Medical and Biological Engineering, 1975, 13, 317.

LEHMANN, U. Short-term and circadian rhythms in the behaviour of the vole, Microtus agrestis (L.). Oecologia, 1976, 23, 185-199.

Moross, G. G., \& Kaufman, G. I. Activity monitor for small animals. Physiology \& Behavior, 1976, 16, 493-495.

Pfister, H. P., Mudge, R. R., \& Harcombe, A. O. A multipurpose activity platform utilized in the open-field setting. Behavior Research Methods \& Instrumentation, 1978, 10, 21-22.

Richter, C. P. Animal behavior and internal drives. Quarterly Review of Biology, 1927, 2, 307-343.

(Received for publication August 2, 1980; revision accepted August 14,1980 .) 